\title{
Reduction of Insertion Resistance by Means of Vibration for Catheters and Endoscopes
}

\author{
Kiyoshi Yoshinaka, Ryo Sakamoto, and Ken Ikeuchi \\ Institute for Frontier Medical Sciences Kyoto University, Kawaharacho 53, \\ Shogoin,sakyo-ku, Kyoto, 6068507, Japan \\ \{yosinaka, ikeuchi\}@frontier.kyoto-u.ac.jp
}

\begin{abstract}
We present a new method to reduce insertion resistance for internal medical instruments by means of vibration. We investigated the relation between frequency of the vibration and the insertion resistance with a pin on flat test system in vitro. And we made a prototype of internal medical probe for in vivo. As a result, vibration is effective to reduce the friction and it is possible to keep a smooth insertion by vibration.
\end{abstract}

\section{Introduction}

It is not always easy to insert medical instruments into a human body without injuring tissues. One of the reasons of difficulty to insert these instruments is friction between organic tissue surface and instruments. Then we present a new method to reduce insertion resistance for internal medical instruments by means of vibration. The decrease of the insertion resistance is necessary for no-invasive and rapid insertion into a human body. If friction controled, a novel high quality medical treatment will be realized and this system will greatly lighten doctors burden.

Therefore this paper shows relationship between insertion resistance and vibration, and presents insertion property of a prototype internal probe with vibration in vivo.

\section{In Vitro and in Vivo Experiments}

We investigated the relation between frequency of the vibration and the insertion resistance with a pin on flat test system in vitro. This experiment system is composed of a linear slider, a vibrating unit, a sensor to measure the insertion resistance and a computer. The computer controls frequency and amplitude of the vibrating unit. Porcine small intestine was used as the test specimen in this experiment.

Next, we made a prototype of internal medical probe shown by Figure.1. This probe is assembled with a vibrating unit, a catheter made of super elastic wire and a capsule. This probe is inserted into a large intestine of a rabbit automatically by the linear actuator and this automated insertion system can measure insertion resistance by the sensor that is installed at the end of catheter. Then we compare the resistance at static insertion with the vibrated insertion. 


\section{Results}

Figure.2 shows that insertion resistance depends on frequency of the vibrating unit, and $90 \mathrm{~Hz}$ of vibration is high enough for effective reduction of resistance.

The result of in vivo experiment shows that when insertion resistance is increased, vibration is effective to reduce the friction, and a smooth insertion is kept with vibration. A 70 percent of insertion resistance is decreased by vibration.

These studies indicate the possibility of friction control of the internal medical instrument by vibration.
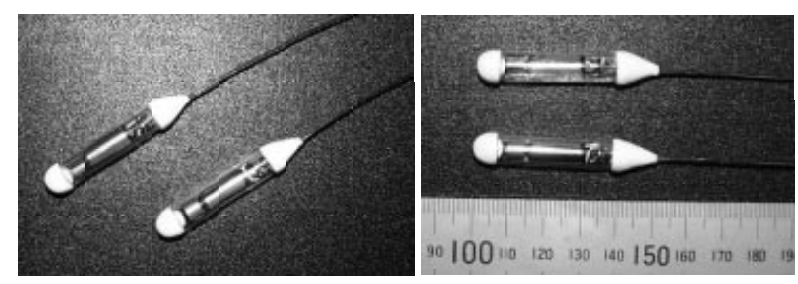

Fig. 1. Apparatus of probes

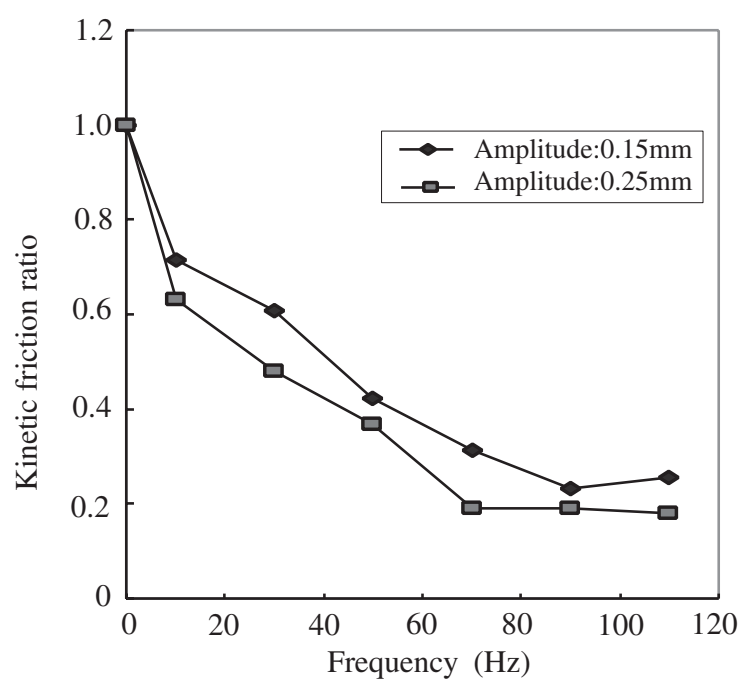

Fig. 2. Effect of vibration amplitude on friction ratio 